\title{
Human Body Specific Absorption Rate Reduction Employing a Compact Magneto-Dielectric AMC Structure for 5G Massive-MIMO Applications
}

\author{
Reza Karimian ${ }^{1}$, Mansoor Dashti Ardakani ${ }^{2, *}$ (D) Shahrokh Ahmadi ${ }^{1}$ and Mona Zaghloul ${ }^{1}$ (D) \\ 1 Department of Electrical Engineering, George Washington University (GWU), Washington, DC 20052, USA; \\ karimian@gwu.edu (R.K.); Ahmadi@gwu.edu (S.A.); zaghloul@gwu.edu (M.Z.) \\ 2 Énergie Matériaux Télécommunications (EMT) Research Centre, Institut National de la Recherche \\ Scientifique (INRS), Université du Québec, Montreal, QC H5A 1K6, Canada \\ * Correspondence: mansoor.dashti@inrs.ca
}

Citation: Karimian, R.; Ardakani, M.D.; Ahmadi, S.; Zaghloul, M. Human Body Specific Absorption Rate Reduction Employing a Compact Magneto-Dielectric AMC Structure for 5G Massive-MIMO Applications. Eng 2021, 2, 501-511. https://doi.org/10.3390/eng2040032

Academic Editor: Antonio Gil Bravo

Received: 30 September 2021

Accepted: 28 October 2021

Published: 4 November 2021

Publisher's Note: MDPI stays neutral with regard to jurisdictional claims in published maps and institutional affiliations.

Copyright: (c) 2021 by the authors. Licensee MDPI, Basel, Switzerland. This article is an open access article distributed under the terms and conditions of the Creative Commons Attribution (CC BY) license (https:// creativecommons.org/licenses/by/ $4.0 /)$.

\begin{abstract}
A compact artificial magnetic conductor (AMC) structure for the application of specific absorption rate (SAR) reduction is presented in this paper. A magneto-dielectric (MD) structure as a host of AMC substrate is used to miniaturize the AMC size. The magneto-dielectric has been designed with a low-profile spiral loop in a way to have a high permittivity and permeability for the desired center frequency of $3.5 \mathrm{GHz}$. Simulation results confirm the zero-degree reflection phase of the proposed AMC unit cell. Moreover, a 70\% reduction has been achieved in comparison to the conventional AMC. To validate the simulation results, a prototype of the board is fabricated and measured with a coplanar waveguide (CPW) antenna for the reflection coefficient. The measurement results display an excellent agreement with the simulation ones. A VOXEL model of a human body is utilized to determine the SAR value of the proposed structure. Considering the maximum SAR value for an average of $10 \mathrm{~g}$ human tissue, more than $70 \%$ SAR reduction is verified for the CPW antenna with the recommended MD-AMC structure compared to a conventional single CPW antenna.
\end{abstract}

Keywords: artificial magnetic conductor (AMC); specific absorption rate (SAR); coplanar waveguide (CPW) antenna; magneto-dielectric (MD); mobile phones; wireless communications

\section{Introduction}

The wireless systems revolution has generated a wave of innovative results that dramatically expand the availability of voice and data almost anywhere. While this change has significantly broadened the possibility for new and better wireless communication ends, it has proffered further considerations for antenna design concepts [1]. Control of specific absorption rate (SAR) is a crucial factor that should be equally considered alongside traditional antenna design parameters. The absorption of electromagnetic energy (EM) emitted from the cellular phone has been considered in recent years. The specific absorption rate is a defined parameter for evaluating power deposition in human tissue. For mobile phone compliance, the SAR value must not exceed the exposure guidelines, 2 watts per kilogram $(\mathrm{W} / \mathrm{kg})$. Mobile handset interaction with the human head has been investigated by numerous published papers providing two types of analysis [2-4].

First is the impact of the human head on the handset performance including the feed-point impedance, gain, and efficiency [5]. Second is the interference of the antenna electromagnetic radiation on the user's head due to the absorbed power, which is measured by predicting the induced SAR in the head tissue [6]. Therefore, many researchers are working on reducing SAR distribution in the human head.

In $[7,8]$, a new platform with a study of attaching conductive materials to the cellular phone body for SAR reduction has been suggested. It is declared that the position of the shielding material is a significant factor for SAR reduction effectiveness [9]. There is a 
requirement to endeavor to reduce the spatial peak SAR in the design phase of the metamaterial because the likelihood of a spatial peak SAR above the recommended exposure limit cannot be entirely ruled out. Another approach is the use of a directional or reflectional antenna [6]. Such an antenna structure sacrifices the availability of signals received from all directions to the phone model.

The further suggestion is the metamaterial structures with virtual control of the material's intrinsic characteristics were employed to reduce the SAR effects $[10,11]$. With proper design and arrangement of meta-cells, stopbands at desired mobile frequencies can be achieved. Although significant SAR reduction was observed with the use of Split Ring Resonators (SRR) meta-cells [12], the antenna profile and fabrication process are severe. The periodicity of SRR is aligned to the direction of mobile EM wave to achieve a low SAR effect. Therefore, the antenna profile size is large. In [13] a perfect electric conductor, the (PEC) reflector was realized between a human head and the driver of a folded loop antenna to achieve this purpose. The result shows that the radiation efficiency is improved, and the peak value of SAR is condensed. While the idea of inserting a PEC between the human head and the mobile antenna is a simple technique that can eliminate the EM wave of the antenna toward the human head, it faces a principal problem of the quarter of wavelength separation requirement.

Among all these approaches, Artificial Magnetic Conductor (AMC) can be an alternative for PEC to achieve our purposes, as it can act as a perfect magnetic conductor (PMC) at particular frequency bands. The AMC surface presents high impedance characteristics in some frequencies and changes the antenna's radiation pattern while maintaining antenna performances such as radiation efficiency and gain even when the antenna is too close to it [14]. In the aforementioned area, several research works have been proposed on AMC as a ground plane of the antenna to reduce RF exposure of mobile phones. However, the size of the designed antenna is relatively significant [12-15].

In this paper, a compact AMC surface, based on a magneto-dielectric substrate with a combination of a coplanar waveguide (CPW) antenna is proposed at $3.5 \mathrm{GHz}$ band to reduce the specific absorption rate. The $3.5 \mathrm{GHz}$ band is essential for Massive MIMO technology and $5 \mathrm{G}$ network strategy to deliver the best customer experience in urban areas [16]. Apart from numerous miniaturization techniques on the patch antenna design [17-19], in this paper, we focused on substrate miniaturization. For this reason, that magneto-dielectric (MD) substrate is a good candidate to achieve this purpose [8]. With the use of MD, an excellent dielectric property with high virtual permittivity can be performed, while the capacitive nature of the patch on the high dielectric substrate can be mitigated due to the magnetic properties (high permeability) of MD. However, most of the meta-substrate used for the antenna design has been employed with metamaterial spiral loops, which makes the whole structure high profile. In this configuration, a novel low-profile MD cell is used as a meta-substrate of a conventional AMC to reduce the total size. Simulation results show that $70 \%$ overall size reduction is obtained with the aforementioned design.

\section{Analysis and Design}

In this section, first, a small printed CPW-fed slot antenna for operation in the $3.5 \mathrm{GHz}$ frequency band has been designed without any metallic layer on the bottom plane of the substrate. The antenna has a simple structure with only one layer of dielectric substrate and metallization. In the proceeding design, a novel AMC structure is described. The CPW antenna and AMC unit cell design simulations are carried out by the frequency solver of CST Microwave Studio and HFSS Software.

\subsection{CPW Antenna Design}

The configuration of the CPW-fed slotted patch antenna is shown in Figure 1. The antenna is printed on a $20-\mathrm{mil}(0.508 \mathrm{~mm})$ thickness RO4003 substrate with the relative permittivity of $\varepsilon_{r}=3.55$. The basic structure of this antenna is a rectangular patch with a dimension of $35 \times 30 \mathrm{~mm}^{2}$. A narrow loop slot is embedded into the patch; the length of 
this slot determines the resonant frequency, and two other parameters, the width of the slot and $l t$, adjust the impedance matching. As obtained by simulation results, the total size of the proposed ground plane has a critical role for impedance matching which is chosen based on HFSS optimization. As there is a linear relationship between the total slot-loop length and the dominant resonant mode, the slot loop length, $l \mathrm{~s}$ is chosen slightly less than one-half of the slot wavelength $(l s=0.42 \lambda s)$; the slot-loop width, $w$ s is kept less than one-quarter of lambda to minimize the magnetic current variation along the loop width.
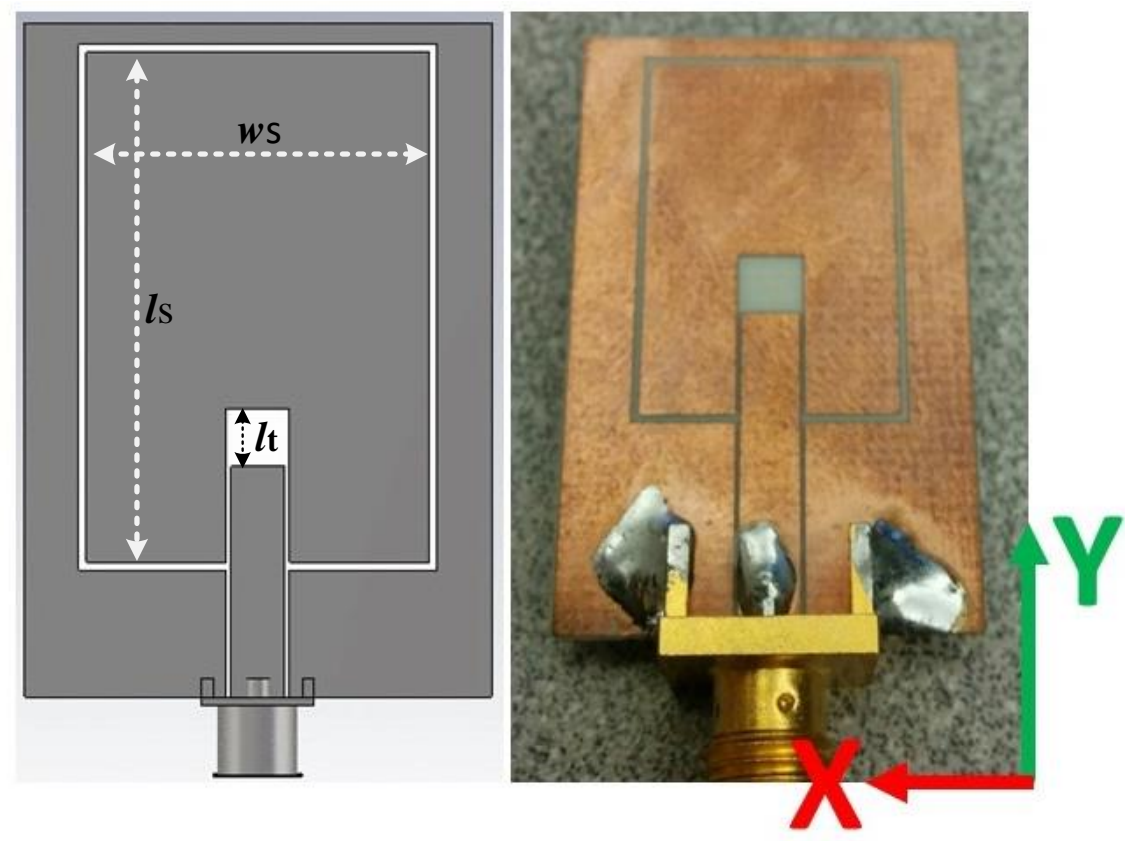

Figure 1. Configuration of the proposed CPW antenna.

However, the slot-loop width is critical for impedance matching. By fixing the optimum parameters of the $\mathrm{CPW}$-fed antenna, proper impedance matching through the operation band for ISM application can be realized. These parameters are shown in Figure 1. The simulated and measured reflection coefficient characteristics of the intended antenna, obtained by CST Software and Agilent E8361C VNA, are shown in Figure 2. An acceptable agreement between simulation and measurement results is obtained.

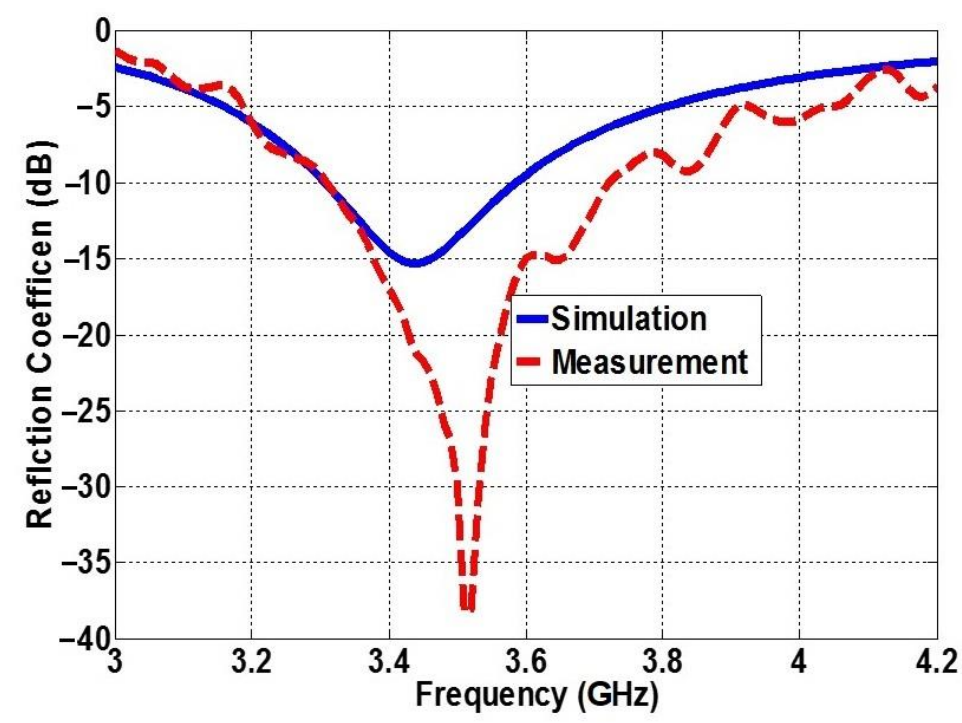

Figure 2. Simulation and measurement results of reflected coefficient of the CPW antenna. 


\subsection{AMC Design}

By definition, an artificial magnetic conductor (AMC) is a structure, which shows a close-to-zero reflection coefficient phase, when it is subjected to the normal TEM plane wave illumination [9]. In their first appearance, AMCs were introduced as the periodic patches shorted to the ground planes via metallic pins [14]. However, they can also be implemented by loading a grounded dielectric substrate with different FSS-type periodic metallization [13]. Numerous techniques were carried out in recent years to compact the AMC unit cell. Some of these include lumped element loading [13] and engineered pattern [9]. However, by changing the permeability and permittivity of the substrate artificially, we have tried to compact the AMC unit cell size. According to [14] the approximate dimensions of the AMC patch can be calculated from the following equations:

$$
\begin{gathered}
L=\mu h \\
C=\frac{W \varepsilon_{0}\left(1+\varepsilon_{r}\right)}{\pi} \cosh ^{-1}\left(\frac{2 W+g}{g}\right)
\end{gathered}
$$

In this model, $W$ and $h$ are the width of the AMC patch and the height of the substrate, respectively. Therefore, as it is cleared from the Equations (1) and (2) by increasing the $\varepsilon$ and $\mu$ of the substrate, $L$ and $C$ increase and the resonance frequency $\left(\omega_{0}=1 / \sqrt{L C}\right)$ moves toward lower frequencies. A magneto-dielectric (MD) structure has been used as a host substrate of a conventional AMC to increase $\varepsilon$ and $\mu$. As the other part of the proposed antenna, the effective medium theory was applied to our MD unit cell to obtain electric permittivity and magnetic permeability. This technique replaces the electromagnetic response of the complicated metamaterial structure with the electromagnetic response of a homogeneous isotropic or anisotropic slab [8]. Cell 1 in Figure 3 depicts the proposed magneto-dielectric substrate. The MD cell consists of metallic strips and vias, which make a loop normal to the $X$-direction. Both host and the upper (for the AMC patch) substrates are Rogers RO4003 with the dielectric permittivity $\varepsilon_{r}=3.55$ and loss tangent $\tan \delta=0.0027$. The Thicknesses of MD and AMC substrates are 32 and 20 mils, respectively. In our proposed MD cell, sidewalls are PMC, and the top and bottom sides are PEC. It is declared that for a plane wave with normal incidence on a homogeneous slab, the wave impedance, and the refractive index are related to the S-parameters as follows:

$$
\begin{aligned}
& S_{11}=\frac{R_{01}\left(1-e^{i 2 n k_{0} d}\right)}{1-R_{01}^{2} e^{i 2 n k_{0} d}} \\
& S_{21}=\frac{\left(1-R_{01}^{2}\right) e^{i 2 n k_{0} d}}{1-R_{01}^{2} e^{i 2 n k_{0} d}}
\end{aligned}
$$

where

$$
R_{01}=\frac{z-1}{z+1}, \& z= \pm \sqrt{\frac{\left(1+S_{11}^{2}\right)-S_{21}^{2}}{\left(1-S_{11}^{2}\right)-S_{21}^{2}}} ;
$$

And by employing the Kramers-Kronig relations according to [20], and by following the formula extraction procedure, the constitutive parameters can be extracted by:

$$
\mu_{e f f}=N_{e f f} Z_{e f f}, \& \varepsilon_{e f f}=\frac{N_{e f f}}{Z_{e f f}}
$$

The extracted constitutive parameters, which are effective permittivity, effective permeability and loss are shown in Figure 4a. In the vicinity of the resonant frequency, the product of the effective permittivity and effective permeability is around 41 . More specifically at the frequency of $3.5 \mathrm{GHz}$, the equivalent, extracted, effective permittivity is $\varepsilon_{e f f}=7.48$ and the equivalent, extracted, effective permeability is $\varepsilon_{\text {eff }}=5.5$. Now with the new $\varepsilon_{\text {eff }}$ and $\mu_{\text {eff }}$ and based on the Equations (1) and (2), the AMC width $(W)$ is calculated. 
Since the width of an AMC patch is larger than the width of an MD unit cell, two MD unit cells are used as the host for our structure. Unit cell 2 in Figure 3 shows the proposed AMC unit cell. Figure $4 \mathrm{~b}$ expresses the reflection phase versus frequency of a unit cell at three hypothetical cases along with the proposed MD-AMC unit cell.

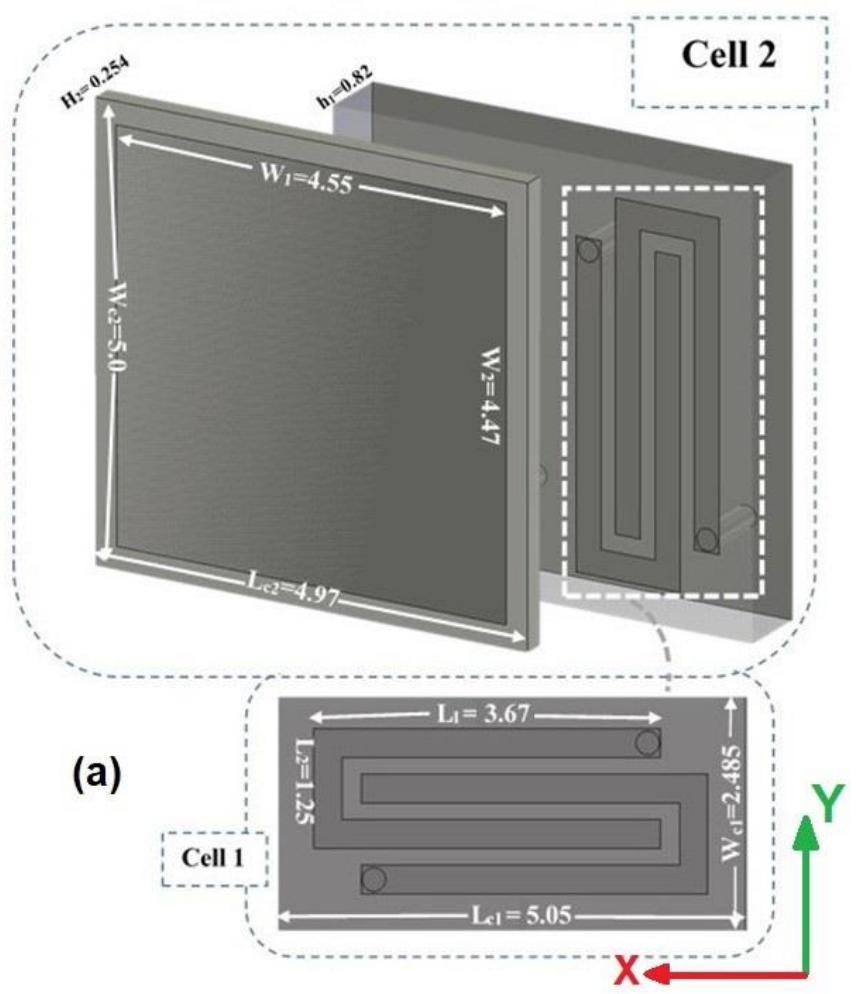

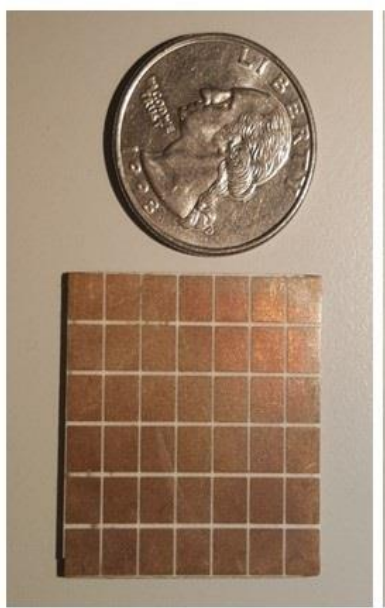

(b)

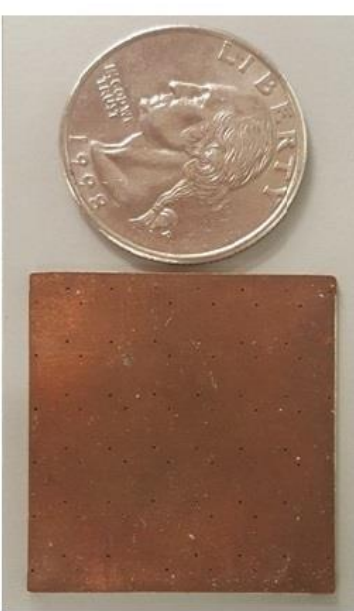

(c)

Figure 3. (a) Magneto Dielectric (MD) unit cell (Cell 1) and MD-AMC unit cell (Cell 2) configuration; The fabricated cells (b) Front view, (c) Back view.

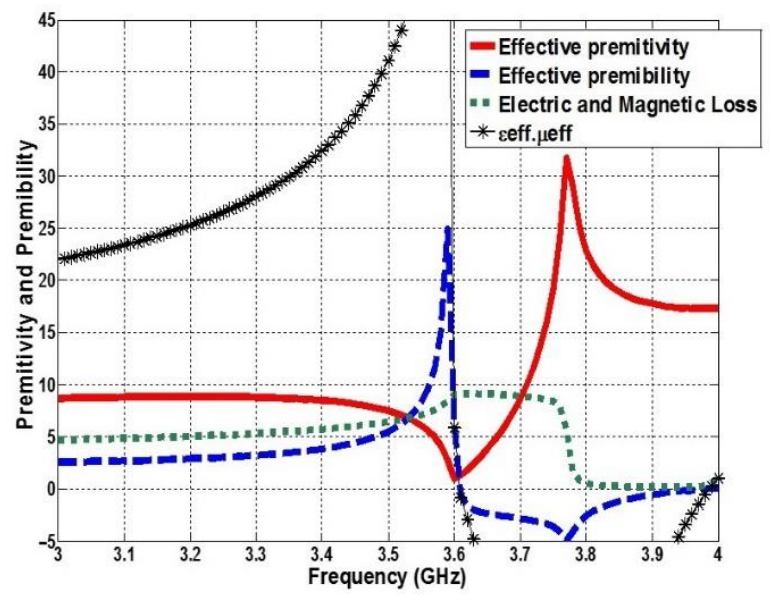

(a)

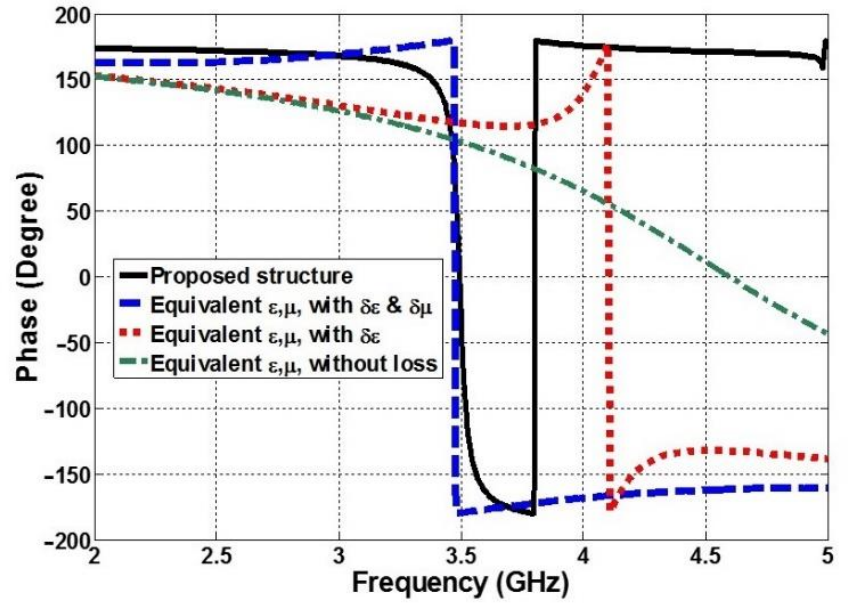

(b)

Figure 4. (a) Effective constitutive parameters of the proposed magneto dielectric substrate. The product of the effective permittivity and permeability is shown by star; (b) Reflection phase comparison between proposed substrate structure with other equivalent constitutive parameters. 
It is evident from the reflection phase that there is a zero phase at the frequency of $3.5 \mathrm{GHz}$ for the proposed MD-AMC cell. To get a better understanding of the MD-AMC cell, three premises were considered to compare with the proposed structure. It is clear from Figure $4 \mathrm{~b}$ that for a substrate with the same $\varepsilon$ and $\mu$ of proposed MD and by considering both electrical and magnetic loss, there will be a good matching between reflection phase responses. There is a shift toward higher frequencies when the magnetic loss is not taken into account. In this case, the electrical loss is around six, which is equal to the whole loss extracted from the proposed MD cell. It is interesting to mention that, even for the previous case in which both losses were considered, the electric loss was dominant. As a result, one can conclude that for the proposed MD cell, the electrical loss is dominant. The last hypothetical is when we have used a substrate with the same $\varepsilon$ and $\mu$ of proposed MD, but without any loss. As we can see, the zero reflection phase has moved toward the upper frequency.

The AMC's bandwidth, on the other hand, has greatly increased. When the reflection phase response of the proposed MD-AMC unit cell is compared to that of a substrate with no loss, it can be concluded that the loss will affect the cell efficiency, which in our instance is the cell bandwidth efficiency. It should be noted that by placing the suggested MD cell precisely beneath a patch antenna, the antenna efficiency and gain will be degraded due to the loss. It does, however, make the structure more compact in our design. By comparison of the reflection phase simulation of a unit cell with the substrate of $\mathrm{RO} 4003$ and the same height of the proposed MD-AMC cell and structure, it is calculated that more than $70 \%$ reduction is achieved by using magneto-dielectric as a host substrate in a conventional AMC.

\section{Results and Discussion}

To validate the performance of the proposed miniaturized AMC, an MD-AMC board is implemented. The CPW antenna is placed $6 \mathrm{~mm}$ above the MD-AMC using Rohacell foam, which has a permittivity of $\varepsilon_{r}=1.006$. Figure 5 shows the prototype of the whole antenna structure in an anechoic chamber. The distance between CPW and MD-AMC board is optimized based on the best performance regarding the reflection coefficient. The comparison between the simulated and measured reflection coefficient of the proposed antenna is depicted in Figure 6. When compared to a single CPW antenna, the simulated result shows a little shift toward higher frequencies, which is more noticeable in the measured result. The difference between the simulation and measured results is mostly due to the foam material between the antenna and MD-AMC that is not entirely similar to air and, also, there is some glue between the antenna and MD-AMC that affects the result. The comparison between simulation and measurement radiation pattern of the proposed antenna for two principle planes $E$ - and $H$-plane is shown in Figure 7, respectively. As it is cleared, the radiation pattern, especially at $E$-field, is similar to a directional antenna which is expected from the MD-AMC board beneath the CPW antenna. The simulation gain result confirms a $3 \mathrm{~dB}$ gain enhancement has been achieved compared to the single CPW antenna without an MD-AMC board. 


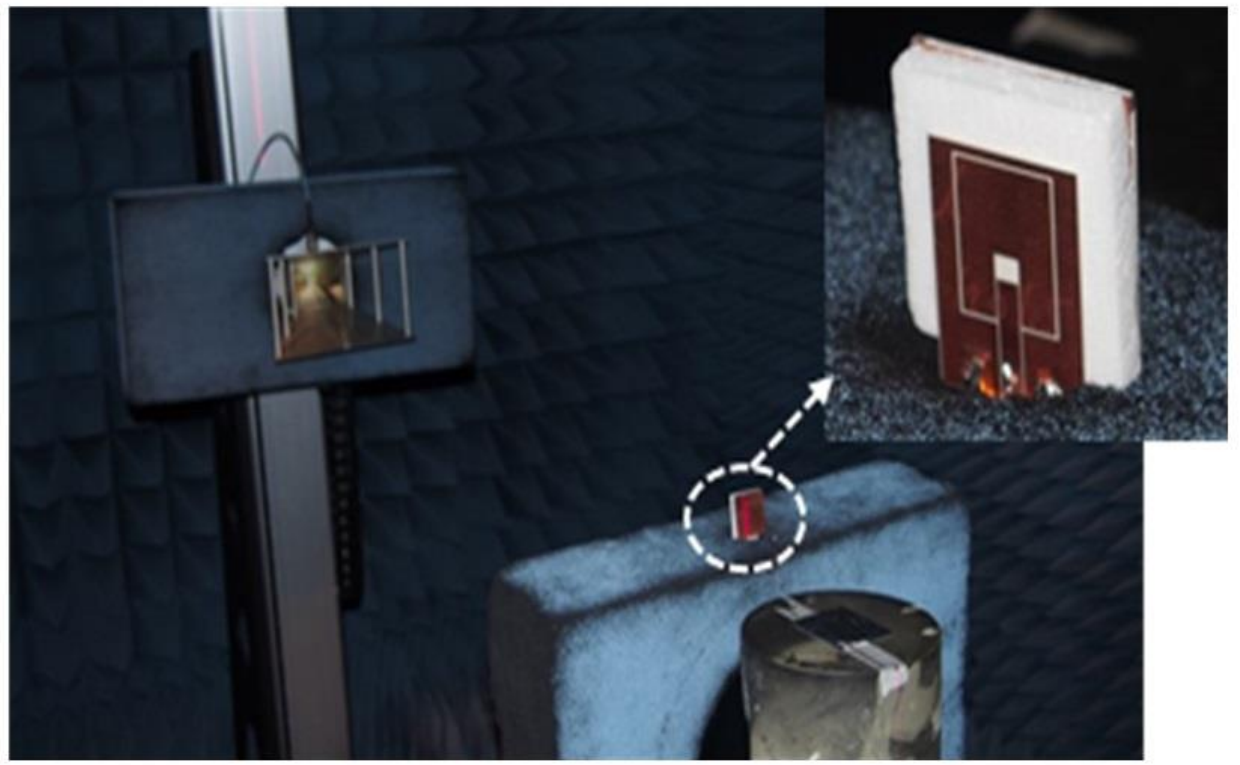

Figure 5. Prototype of the fabricated CPW antenna above proposed MD-AMC substrate in an anechoic chamber.

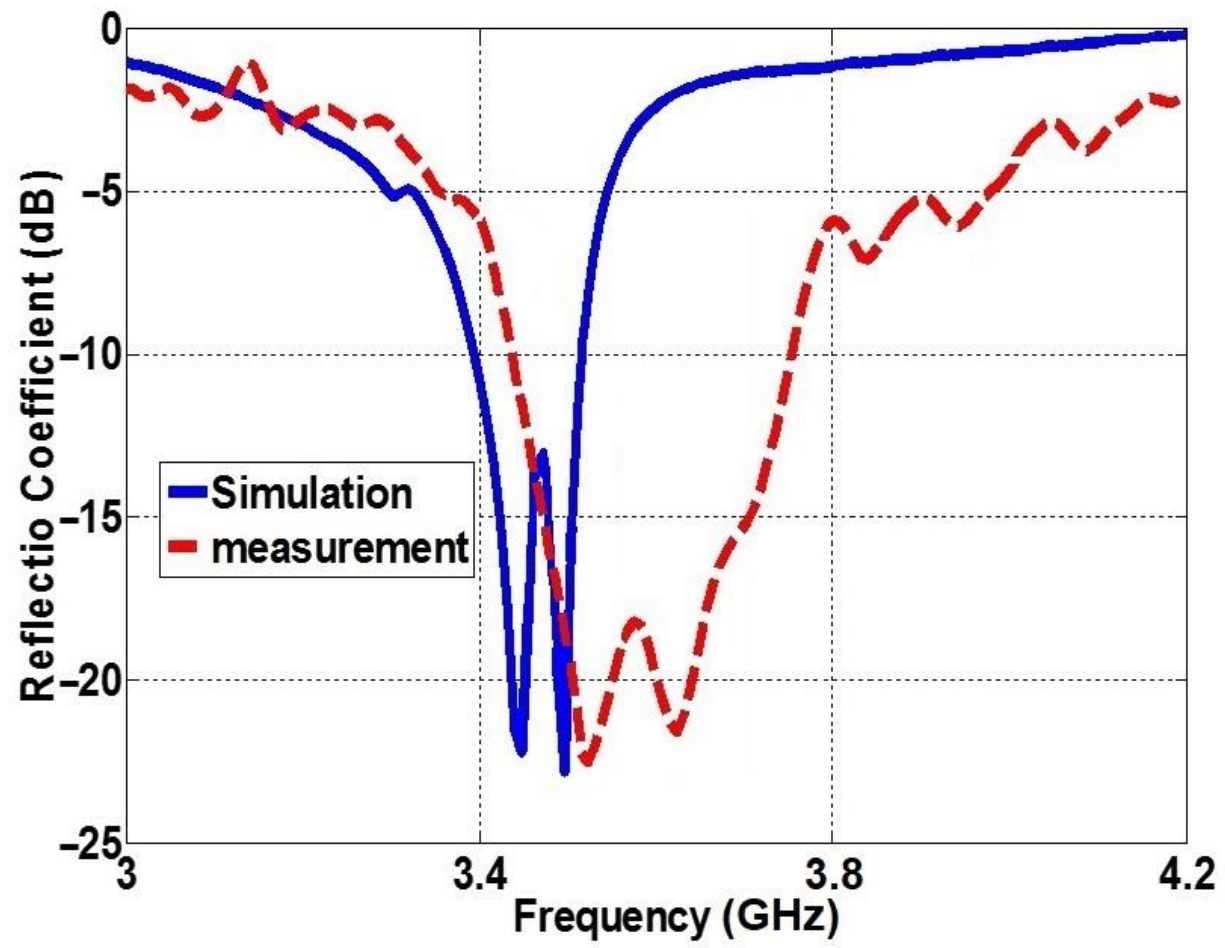

Figure 6. Simulation and measurement results of reflected coefficient of the CPW antenna above MD-AMC substrate. 


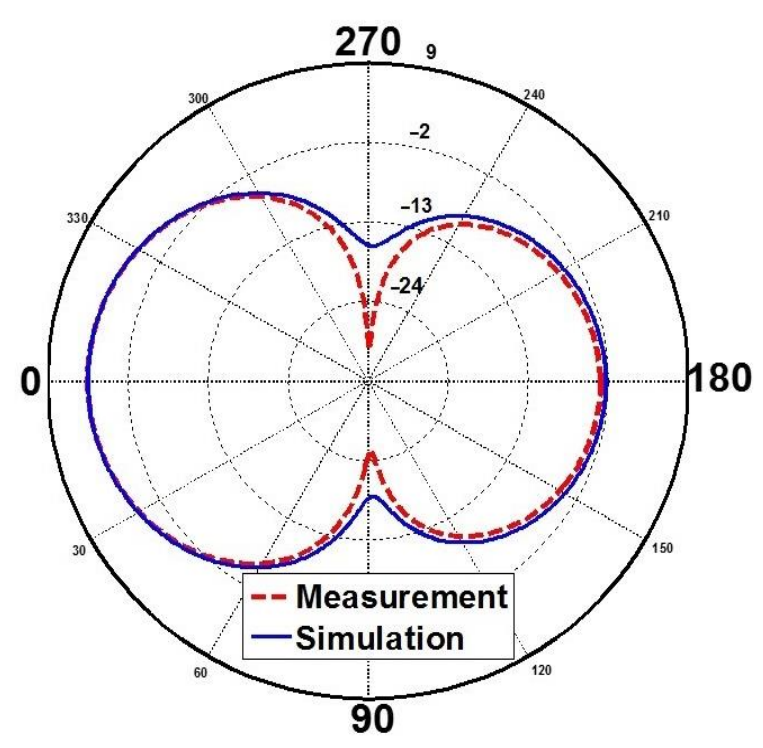

(a)

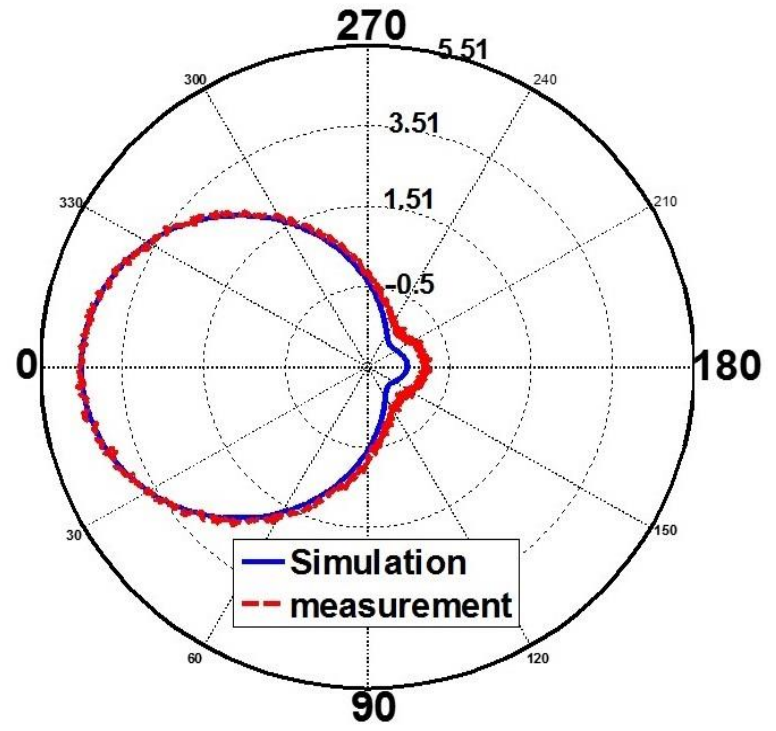

(b)

Figure 7. Simulation and measurement results of the radiation pattern of the CPW antenna over proposed MD-AMC board for (a) E-plane, and (b) H-plane.

\section{SAR Analysis}

Specific Absorption Rate (SAR) is a crucial factor to measure the maximum absorption rate by human tissues when exposed to the radiofrequency electromagnetic field. The SAR value according to IEEE Standards Association (IEEE C95.1-2019) and EU standards should not exceed $2 \mathrm{~W} / \mathrm{kg}$. From the electric field within the tissues, SAR can be calculated as follow:

$$
\operatorname{SAR}=\frac{\sigma|E|^{2}}{\rho}
$$

where $\sigma$ is the conductivity in the tissue in $\mathrm{S} / \mathrm{m}, E$ is the $\mathrm{r}$ oot mean square (RMS) electric field in $\mathrm{V} / \mathrm{m}$ and $p$ is the mass density of the tissue in $\mathrm{kg} / \mathrm{m}^{3}$. To calculate the SAR value of the proposed antenna, a voxel model was utilized. Figure 8 , shows the set-up simulation model that is used in the simulation. The original model of the VOXEL is derived from high-resolution CT data segmented into 125 tissue types. The model has a resolution of $3.6 \mathrm{~mm}^{3}$. For a better understanding of the proposed structure effectiveness regarding SAR, two separate simulations were carried out. At the first simulation, the antenna above the MD-AMC board was placed with a distance of $7 \mathrm{~mm}$ of the model head, and for the other simulation, the CPW antenna without the MD-AMC board and with the same distance of another simulation was placed near the human head. The SAR distribution for both cases is simulated and shown in Figure 8. The maximum SAR value over the average mass of $10 \mathrm{~g}$ tissue is $0.87 \mathrm{~W} / \mathrm{kg}$ for a single CPW antenna and $0.26 \mathrm{~W} / \mathrm{kg}$ for the proposed structure. As a result, more than $70 \%$ reduction has been achieved with our proposed MD-AMC board. In addition to a geographic SAR distribution in Figure 8, the specific point value of the SAR is depicted in Figure $9 a, b$ for an antenna with MD-AMC board and antenna without MD-AMC board, respectively. For the two latter figures center of the CPW antenna is the origin of the coordinate. As realized, the maximum SAR is for the coordinate of $X<0$ and $Y=0$. It is expected to have zero SAR for the space between the antenna and the VOXEL model. Consequently, by moving toward negative $\mathrm{X}$-direction, the value of the SAR will decrease. 


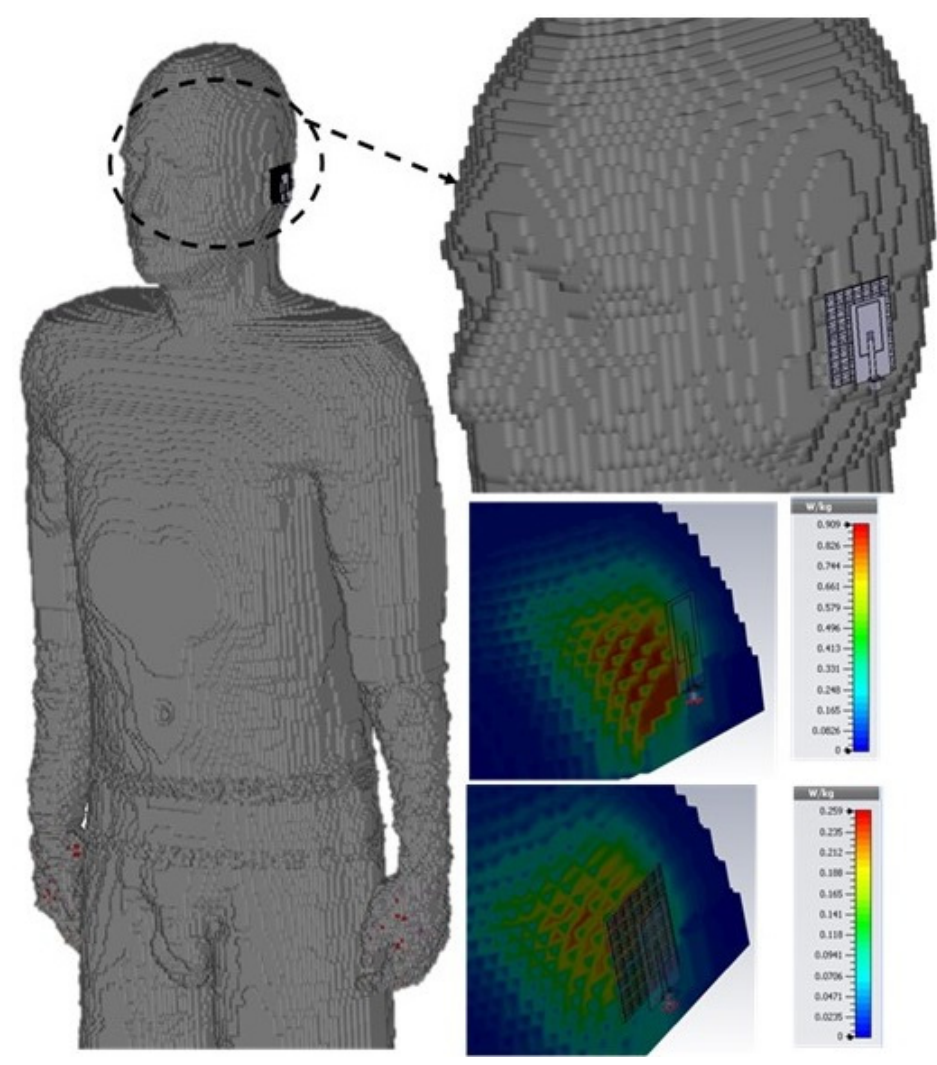

Figure 8. 3-D model of SAR calculator set-up that consists of a Voxel model, the antenna close to the head and ear of the body, and simulated SAR results for CPW without MD-AMC board (top) and CPW with MD-AMC board (bottom).

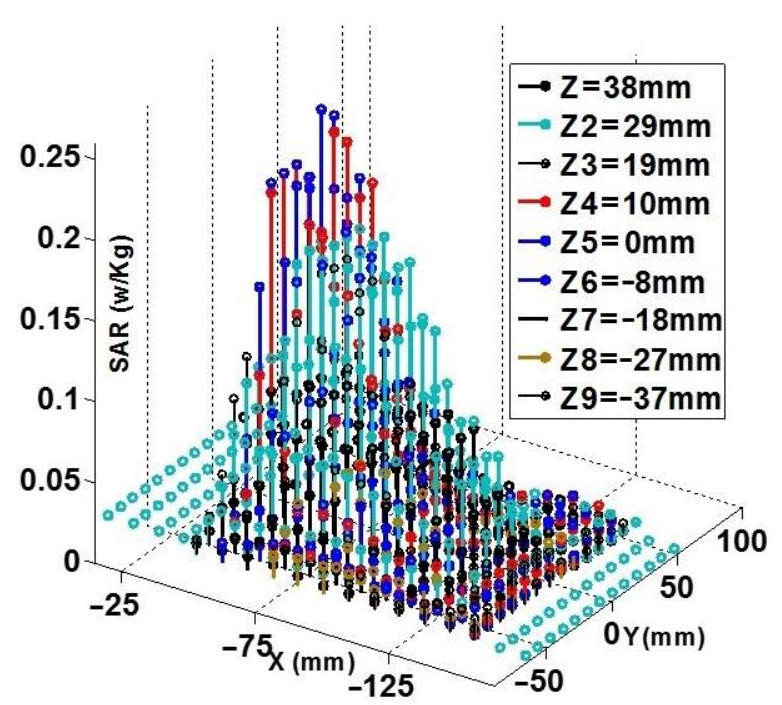

(a)

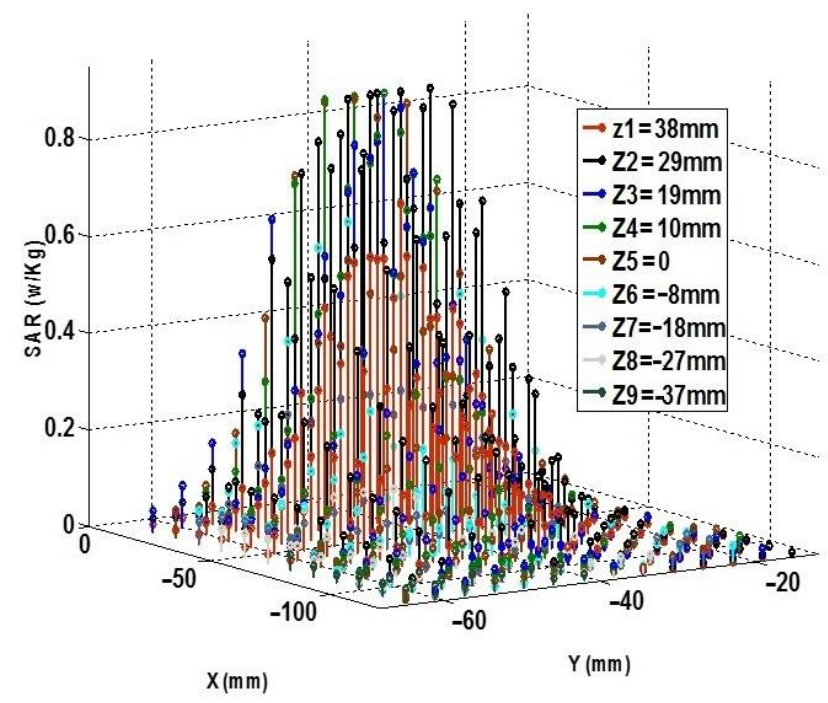

(b)

Figure 9. Simulation results of SAR distribution (a) with MD-AMC board, (b) without MD-AMC board.

\section{Conclusions}

Design, simulation, and implementation of a compact artificial magnetic conductor based on a magneto-dielectric substrate has been proposed in this paper to reduce the SAR significantly. To miniaturize a conventional AMC unit-cell, a spiral loop including strip patches and vias are inserted beneath the AMC cell as a host dielectric, which turns a 
simple substrate with $\left(\varepsilon_{r}=3.5, \mu=1\right)$ into an artificial substrate with $\left(\varepsilon_{\text {eff }}=7.48, \mu_{\text {eff }}=5.5\right)$. The reflection phase simulation result of the proposed AMC unit cell shows that a 70\% reduction has been achieved. To validate the MD-AMC board, a prototype was fabricated and measured with a simple CPW antenna. The measurement result of the reflected coefficient of the antenna shows that it has been followed by simulation one. Finally, a VOXEL model was used to set up a model to calculate the SAR value of the proposed structure. The simulation result shows that more than $70 \%$ reduction has been achieved regarding SAR compared to a simple CPW antenna. High efficiency, proper matching, low SAR value and compact structure make this structure a good candidate for biomedical and massive MIMO application.

Author Contributions: Conceptualization, R.K.; methodology, R.K. and M.D.A.; software, R.K. and M.D.A.; validation, R.K., M.D.A.; formal analysis, R.K.; investigation, M.D.A.; resources, M.D.A.; data curation, R.K.; writing—original draft preparation, R.K.; writing—review and editing, M.D.A.; visualization, M.D.A.; supervision, S.A. and M.Z.; project administration, R.K.; funding acquisition, S.A. and M.Z. All authors have read and agreed to the published version of the manuscript.

Funding: This research received no external funding.

Institutional Review Board Statement: Not Applicable.

Informed Consent Statement: Not Applicable.

Data Availability Statement: Not Applicable.

Conflicts of Interest: The authors declare no conflict of interest.

\section{References}

1. Ardakani, M.D.; Tabatabaefar, M. A transparent robust quasi-isotropic circularly polarized antenna for Cub-Sat and outdoor wireless. Eng. Rep. 2020, 2, e12224. [CrossRef]

2. Faruque, M.R.I.; Islam1, M.T.; Misran, N. Design Analysis of new Metamaterial for EM Absorption Reduction. Prog. Electromagn. Res. 2012, 124, 119-135. [CrossRef]

3. Dashti Ardakani, M.; Farahani, M.; Akbari, M.; Tatu, S.O. A Compact Wideband Cubic Dielectric Resonator Antenna for Integrated 60-GHz MHMIC Short-range Transceivers. In Proceedings of the 2020 IEEE International Symposium on Antennas and Propagation and North American Radio Science Meeting, Montreal, QC, Canada, 5-10 July 2020; pp. 71-72.

4. Karimian, R.; Taravati, S.; Ardakani, M.D.; Ahmadi, S.; Zaghloul, M. Nonreciprocal-Beam Phased-Array Antennas Based on Transistor-Loaded Phase Shifters. IEEE Trans. Antennas Propag. 2021. [CrossRef]

5. Duncombe, J.U. Infrared Navigation-Part I: An Assessment of Feasibility. IEEE Trans. Electron. Devices 1959, ED-11, 34-39.

6. Kuo, C.-M.; Kuo, C.-W. SAR distribution and temperature increase in the human head for mobile communication. In Proceedings of the Antennas and Propagation Society International Symposium, Columbus, OH, USA, 22-27 June 2003; IEEE: Columbus, OH, USA, 2003; Volume 2, pp. 1025-1028.

7. Karimian, R.; Pourahmadazar, J.; Nedil, M.; Denidni, T.A. On the design of low SAR CPW antenna with magneto dielectric AMC based ground plane. In Proceedings of the 2016 10th European Conference on Antennas and Propagation (EuCAP), Davos, Switzerland, 10-15 April 2016; pp. 1-5.

8. Tay, R.Y.S.; Balzano, Q.; Kuster, N. Dipole configurations with strongly improved radiation efficiency for hand-held transceivers. IEEE Trans. Antennas Propag. 1998, 46, 798-806. [CrossRef]

9. Karimian, R.; Ardakani, M.D.; Ahmadi, S.; Zaghloul, M. High resolution beam switch antenna based on modified CRLH Butler matrix. Eng. Rep. 2021, 3, e12287.

10. Ardakani, M.D.; Amiri, R. Mutual coupling reduction of closely spaced MIMO antenna using frequency selective surface based on metamaterials. Appl. Comput. Electromagn. Soc. (ACES) J. 2017, 32, 1064-1068.

11. Pinto, Y.; Begnaud, X. Mobile phone model with metamaterials to reduce the exposure. J. Appl. Phys. A 2016, 122, 1-7. [CrossRef]

12. Karimian, R.; Tadayon, H. Compact ultra-wideband antenna with band-notched based on defected ground structure. J. Eng. 2014, 2014, 30-31. [CrossRef]

13. Shynu, S.V.; Ammann, M.J. A printed CPW-fed slot-loop antenna with narrowband omnidirectional features. IET Microw. Antennas Propag. 2009, 3, 673-680. [CrossRef]

14. Olmos-Trigo, J.; Sanz-Fernández, C.; Abujetas, D.R.; Lasa-Alonso, J.; de Sousa, N.; García-Etxarri, A.; Sánchez-Gil, J.A.; MolinaTerriza, G.; Sáenz, J.J. Kerker Conditions upon Lossless, Absorption, and Optical Gain Regimes. Phys. Rev. Lett. 2020, $125,073205$. [CrossRef] [PubMed]

15. Bag, A.; Neugebauer, M.; Wozniak, P.; Leuchs, G.; Banzer, P. Transverse Kerker Scattering for Angstrom Localization of Nanoparticles. Phys. Rev. Lett. 2018, 121, 193902. [CrossRef] 
16. Chen, X.; Grzegorczyk, T.M.; Wu, B.I.; Pacheco, J.; Kong, J.A. Robust method to retrieve the effective constitutive parameters of metamaterials. Phys. Rev. E Stat. Phys. Plasmas Fluids Relat. Interdiscip. Top. 2004, 70, 016608. [CrossRef] [PubMed]

17. Ojaroudi Parchin, N.; Jahanbakhsh Basherlou, H.; Alibakhshikenari, M.; Ojaroudi Parchin, Y.; Al-Yasir, Y.I.; Abd-Alhameed, R.A.; Limiti, E. Mobile-Phone Antenna Array with Diamond-Ring Slot Elements for 5G Massive MIMO Systems. Electronics 2019, 8 , 521. [CrossRef]

18. Ardakani, M.D.; Pourahmadazar, J.; Tatu, S.O. A monopole antenna with notch-frequency function for UWB application. In Proceedings of the 2017 XXXIInd General Assembly and Scientific Symposium of the Internat. Union of Radio Science (URSI GASS), Montreal, QC, Canada, 19-26 August 2017; pp. 1-4.

19. Karimian, R.; Ardakani, M.D.; Koosha, B.; Ahmadi, S.; Zaghloul, M. A Compact Beam Steering Dielectric Resonator Antenna for Wireless Power Transfer. In Proceedings of the 2021 United States National Committee of URSI National Radio Science Meeting (USNC-URSI NRSM), Boulder, CO, USA, 4-9 January 2021; pp. 5-6.

20. Szabo, Z.; Park, G.H.; Hedge, R.; Li, E.P. A Unique Extraction of Metamaterial Parameters Based on Kramers Kronig Relationship. IEEE Trans. Microw. Theory Tech. 2010, 58, 2646-2653. [CrossRef] 